\title{
In reply: Anesthesia bronchoscopes and CSA Standard Z314.8-14
}

\author{
Gale Schultz, RN, BN
}

Received: 30 March 2017/Revised: 12 April 2017/Accepted: 18 April 2017/Published online: 4 May 2017

(C) Canadian Anesthesiologists' Society 2017

Thank you for the concerns you raised ${ }^{1}$ about evidence to support the Clause 11.8.1 informative notes and one special requirement in Table 1 in Canadian Standards Association (CSA) Z314.8-14 Decontamination of reusable medical devices (may be purchased at http://shop.csa.ca/en/canada/ sterilization/z3148-14/invt/27010632014).

It is important to be aware that, in a CSA standard, notes are an informative accompaniment to the mandatory requirement that is written in the preceding clause or subclause. In this case, the preceding Clause 11.7.1.2 requires that the reprocessing of each type of endoscope shall be consistent with the manufacturer's validated reprocessing instructions, including procedures for drying, transporting it to a storage area, and storage. The purposes of the following notes are to separate any explanatory information from the actual normative requirement and provide additional direction or guidance for the user. It is purely informative material that helps to clarify further the meaning behind the (sub)clause. As such, it is not meant as an alternative requirement for users to follow.

\subsubsection{Note (b)}

There is little differentiation in the various standards between the risk of patient infection by flexible scopes used in the upper respiratory tract for airway management and those used in the lower respiratory tract for medical interventions. In addition, there is no consensus in the

G. Schultz, RN, BN ( $₫)$

MDR, WRHA, Winnipeg, Canada

e-mail: gschultz@wrha.mb.ca literature or among other standard-setting bodies regarding the need for bronchoscopes to be sterilized $v s$ being highlevel disinfected when it comes to microbial growth.

\subsubsection{Note (c)}

This informative note references an article published by the Gastroenterological Society of Australia. ${ }^{2}$ In that same article, the authors elaborated further to state that "extended storage is only permitted if recent (within 12 months) routine microbiological surveillance of the endoscope has shown negative culture results," which allows for the possibility of extended storage under certain specified conditions. Once again, this note is not meant to be a mandatory requirement for users to reprocess bronchoscopes that have been stored $12 \mathrm{hr}$ or more since their last reprocessing.

\subsubsection{CSA special requirement}

In response to Alberta Health Services' practice of preassembling bronchoscopes for emergency use, storage of preassembled bronchoscopes is not recommended by the manufacturer's instructions for use or advisory bodies such as the Gastroenterological Society of Australia/Society of Gastroenterology Nurses \& Associates, Public Health Agency of Canada. For storage requirements, users should always refer to the manufacturer's reprocessing and storage instructions, as stated in Clause 11.7.1.2.

In closing, our response to this letter is to highlight that consensus-based standards are written in the clearest language possible to try to minimize misinterpretation of 
the meaning behind the mandatory requirements. They are, however, living documents and can be revised as needed.

The notes that provide additional information following clauses or subclauses provide a division of the requirements separate from the explanatory information that users may or may not choose to read, agree with, or follow.

To submit recommendations for changes with rationale to the CSA Z314.8-14 standard, please contact:

\section{Gale Schultz RN BN}

WRHA Regional Director, MDR

CSA Technical Advisory Committee Co-chair

E-mail: gschultz@wrha.mb.ca

Conflicts of interest None declared.
Editorial responsibility This submission was handled by Dr. Gregory L. Bryson, Deputy Editor-in-Chief, Canadian Journal of Anesthesia.

\section{References}

1. Walker A, Coutts M, Davies JM. Anesthesia bronchoscopes and CSA Standard Z314.8-14. Can J Anesth 2017; 64: this issue. DOI:10.1007/s12630-017-0890-4

2. Gastroenterological Society of Australia and Gastroenterological Nurses College of Australia Clinical Update - Infection Control in Endoscopy - Third Edition, 2010. Mulgrave, Victoria. Available from URL: http://membes.gesa.org.au/membes/files/Clinical\%20 Guidelines\%20and\%20Updates/Infection_Control_in_Endoscopy_ Guidelines_2014.pdf (accessed April 2017). 